\title{
Quantificação semiautomática da velocidade do sangue em imagens de ultrassonografia Doppler
}

\author{
Mauricio Higa, Paulo Eduardo Pilon, Sílvia Helena Gelás Lage, Marco Antonio Gutierrez*
}

Resumo Imagens de gráfico de velocidade sanguínea adquiridas pela ultrassonografia Doppler podem ser utilizadas para investigar possíveis relações entre as informações presentes nas mesmas e a ocorrência de doenças cardiovasculares, entre outras aplicações. Com o objetivo de facilitar este tipo de estudo em protocolos clínicos envolvendo centenas de pacientes, um aplicativo computacional foi desenvolvido para extrair dados quantitativos daqueles gráficos. Após uma etapa de calibração e seleção da região de interesse definida pelo usuário, o algoritmo automaticamente detecta o eixo de referência e a envoltória da curva, viabilizando o cálculo dos picos de velocidade e a integral de velocidade e tempo (VTI). Técnicas de processamento de imagem, tais como Componentes Conexos e filtros Gaussiano e Mediano, foram aplicadas para melhorar a robustez da detecção da envoltória. Uma análise comparativa entre as medições obtidas pelos sistemas de ultrassonografia comerciais operados por especialistas (denominadas como " $A$ ") e pela presente metodologia (denominadas como " $B$ ”) incluiu velocidades de pico sistólico e VTIs das artérias carótida comum e braquial na condição basal, da braquial durante a resposta de hiperemia reativa e de exames ecocardiográficos. Os resultados apresentaram uma pequena diferença média, calculada como $d=\overline{A-B}$, pela análise de BlandAltman e uma alta correlação tanto para as velocidades de pico $(\mathrm{d}=0,02 \mathrm{~m} / \mathrm{s} ; \mathrm{r}>0,99 ; \mathrm{p}<0,01 ; 102$ amostras $)$ quanto para as VTIs $(\mathrm{d}=1,25 \mathrm{~cm} ; \mathrm{r}>0,99 ; \mathrm{p}<0,01 ; 75$ amostras). Estes resultados indicam a confiabilidade desta metodologia que, complementada pela interface gráfica amigável do aplicativo desenvolvido, pode ser facilmente utilizada pelos clínicos para seus estudos em larga escala baseados nas imagens Doppler ultrassonográficas.

Palavras-chave Ultrassonografia Doppler, Gráfico de velocidade do sangue, Fluxo de sangue, Integral de velocidade e tempo, Detecção da envoltória.

\section{Semi-automatic quantification of blood velocity in Doppler ultrasound images}

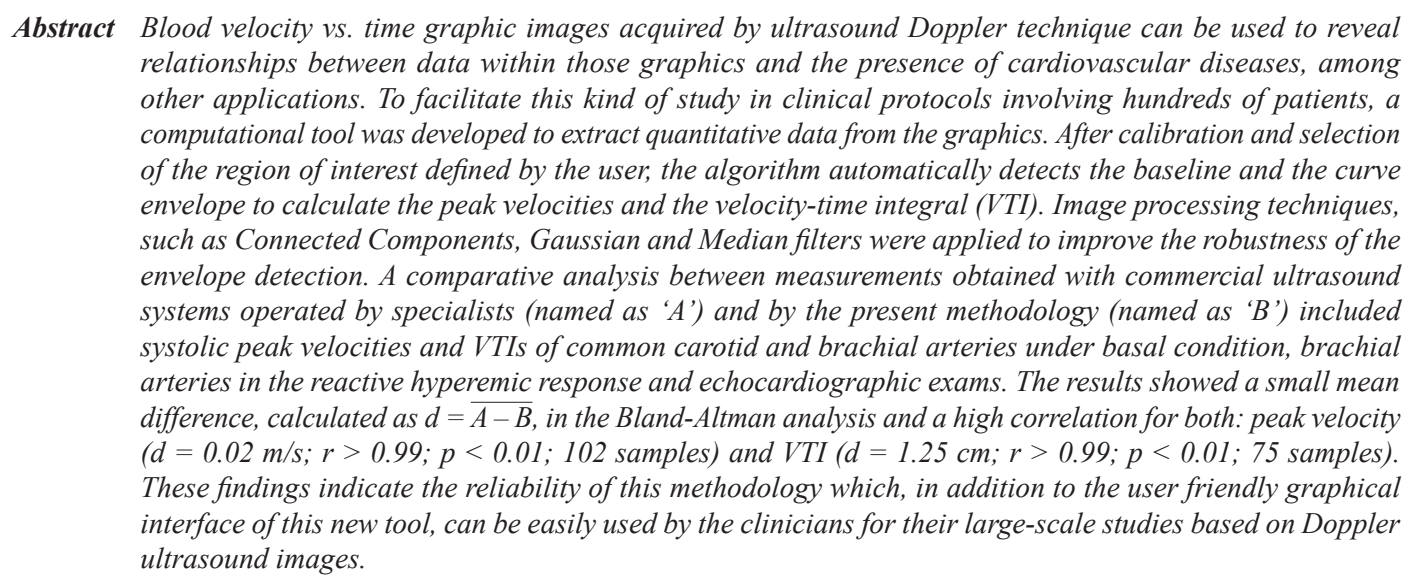

Keywords Doppler ultrasound, Blood velocity, Blood flow, Velocity-time integral, Envelope detection.

*e-mail: marco.gutierrez@incor.usp.br 


\section{Extended Abstract}

\section{Introduction}

Ultrasound signals have been extensively used in clinical sites, by exploiting Doppler effect to measure vascular blood velocity and flow, among other applications (Gerhard-Herman et al., 2006; Nichols and O'Rourke, 1990). Typically, a spectrum of frequencies related to the different velocities of the blood cells (Equation 1) is presented as a curve of velocity vs. time (Figure 1). This information can reveal important relationships between the frequency spectral pattern along the cardiac cycle and the presence of cardiovascular diseases (Hoskins, 1990; Yao, 2004), among other examples (Carvalho et al., 2006; Corretti et al., 2002; Lage et al., 2002).

Since commercial ultrasound systems are primarily dedicated to get instantaneous data for individual patients, they have, in general, low flexibility to perform large-scale researches. Thus, to facilitate this kind of study in clinical protocols involving hundreds of patients, a user friendly computational tool was developed to extract quantitative data from spectral display of Doppler images.

The aim of this paper is to describe and evaluate the semiautomatic methodology implemented in the tool to detect the spectrum envelope and to measure blood velocity, flow and velocity-time integral (VTI).

\section{Material and Methods}

The spectral images displayed in the form of blood velocity vs. time graphics were acquired from four different ultrasound system models: Apogee 800 Plus, Cypress ${ }^{T M}$, HDI 5000, and Sonos 5500.

To extract blood velocity and flow from the images above, a new module was added to a previously developed computational tool (Gutierrez et al., 2002; Pilon, 2002) which, given B-mode ultrasound images as input data, was specialized for arterial wall thickness and lumen diameter measurements.

An overview of the methodology used in this new module is presented below.

After two operator dependent steps: calibration and selection of the region of interest (ROI) (Figure 2), a Gaussian filter is applied to the grayscale input image to attenuate high frequency noise (Figure 3).

The detection process of the time axis $(x)$ considers that it is exactly in the horizontal direction and their pixel intensities have small variation. Thus, Equations 2, 3 and 4 calculates the ordinate ' $y$ ' expected for the axis ' $x$ ', which will be the reference $(0 \mathrm{~m} / \mathrm{s})$ to the velocity calculation.

The image is, then, converted to binary values depending on a threshold level (default: 40) that can be adjusted by the user (Figure 5). A Median filter (size: $3 \times 3$ pixels) is applied to the binary image for edge smoothing and spurious suppression (Figure 6).

The envelope detection step is initialized with two horizontal lines: one at the top and the other at the bottom of the ROI. Each point of these lines is moved down or up to the border of the binary curve. At last, the algorithm holds either the superior or the inferior contour (Figure 4), assuming that, in general, the desired one has higher amplitude variation.

Finally, after peak detections, the algorithm computes the mean peak velocity, the mean envelope velocity and the VTI according to the Equations 5, 6 and 7, respectively. In addition, if $B$-Mode images are available, an arterial wall interface detection module determines the vessel diameter and the blood flow can be estimated using 8 and 9 .

A comparative analysis between commercial ultrasound systems operated by specialists and the present methodology included systolic peak velocities (102 samples) and VTIs (75 samples) of common carotid and brachial arteries under basal condition, brachial arteries in the reactive hyperemic response and echocardiographic exams (Table 1).

\section{Results and Discussion}

Figure 8 shows Bland-Altman's (Bland and Altman, 1986) and Linear Regression graphics for the systolic peak velocity analysis, where 'A' refers to the measurements done with a commercial ultrasound system and ' $B$ ' refers to the proposed methodology. Mean difference, calculated as $d=\overline{A-B}$, standarddeviation, correlation coefficient, and linear equation results are presented in Table 2. Similarly, Figure 9 and Table 3 were obtained for VTI's analysis.

The small mean difference and high correlation for both, peak velocity and VTI, indicate the reliability of this methodology and these findings are better than the results presented by Tschirren et al. (2001).

Visual results showing the envelopes drawn on the blood velocity graphics point that, by using the proposed methodology (Figure $4)$, the envelope line is more refined than that obtained by manual operation of an ultrasound system (Figure 1), because in the latter, the user does not notice or, simply, disregards small image brightness variations, which means that this procedure is highly dependent on the user's subjective evaluation and it is hardly reproducible.

\section{Conclusion}

By processing diversity of common carotid, brachial and echocardiographic Doppler image samples collected from four different commercial ultrasound systems, the methodology implemented in this tool to measure velocity and VTI was validated by the Bland-Altman's analysis and by the correlation coefficient. Visual analysis also confirmed that the spectrum envelope detection is very satisfactory.

The user friendly graphical interface combined to the semiautomatic characteristic of this tool intends to help the clinicians for their studies based on Doppler ultrasound images, with the following advantages: to save operational time, to lower subjective results, and to support measurement reproducibility. 


\section{Introdução}

Baseada na diferença entre as frequências de um sinal transmitido e de um sinal recebido quando há um movimento relativo entre a fonte e o receptor destes sinais, a técnica Doppler, a partir de sinais de ultrassom, tem sido extensivamente empregada em ambientes clínicos para a medição da velocidade e do fluxo de sangue vascular, entre outras aplicações (Gerhard-Herman et al., 2006; Nichols e O'Rourke, 1990).

Assumindo, inicialmente, que um sinal mecânico de frequência $f_{0}$, gerado por um transdutor, se propaga pelo tecido biológico em uma direção tal que o seu vetor forme um ângulo $\theta$ com o vetor de velocidade $v$ de uma célula vermelha do sangue, admitindo também que a velocidade de propagação no tecido mole $(c \approx 1.540 \mathrm{~m} / \mathrm{s})$ é muito superior à velocidade da célula ( $v$, da ordem de grandeza de $1 \mathrm{~m} / \mathrm{s}$ ). Pelo efeito Doppler o sinal refletido por esta célula e que retorna ao transdutor é composto por outra frequência $f$ e a diferença entre as frequências possibilita o cálculo da velocidade da célula, segundo a expressão (Bamber e Tristam, 1993; Routh, 1996; Yao, 2004):

$v=\frac{c\left(f-f_{0}\right)}{2 f_{0} \cos \theta}$

Para um conjunto de células que constituem o fluxo sanguíneo e que espalham e refletem o sinal de ultrassom em frequências distintas devido às suas velocidades distintas, um gráfico de velocidade em função do tempo não é composto por uma linha simples e bem definida, mas por um espectro de frequências correspondentes às diferentes velocidades das células a cada unidade de tempo (Figura 1). Este gráfico contém informações que podem indicar possíveis relações entre a forma espectral durante o ciclo cardíaco e a ocorrência de doenças cardiovasculares (Hoskins, 1990; Yao, 2004). Além disso, existem estudos que requerem o conhecimento da velocidade e fluxo sanguíneos para a obtenção da disfunção endotelial na insuficiência cardíaca (Carvalho et al., 2006; Corretti et al., 2002) e aqueles que visam monitorar os efeitos da administração de determinados medicamentos no sistema circulatório (Lage et al., 2002).

A extração de dados quantitativos de velocidade do sangue utilizando os sistemas comerciais de ultrassonografia apresenta pouca flexibilidade quando se deseja realizar estudos em larga escala, com uma série de pacientes, para correlacionar estas medidas com alguma possível doença, visto que estes sistemas são dedicados principalmente à execução da rotina de exames para prover informações instantâneas, individualmente.
O objetivo deste artigo é descrever e avaliar uma metodologia semiautomática para a detecção da envoltória do espectro do gráfico de velocidade e o cálculo de velocidades (média e média dos picos), fluxos (média e média dos picos) e a integral de velocidade multiplicada pelo tempo (velocity-time integral - VTI) de imagens Doppler ultrassonográficas.

\section{Materiais e Métodos}

\section{Sistemas de ultrassonografia}

As imagens que contêm os espectros representados pelas curvas de velocidade em função do tempo utilizadas neste trabalho foram adquiridas pela ultrassonografia Doppler de quatro modelos distintos de sistemas pertencentes ao Instituto do Coração do Hospital das Clínicas da Faculdade de Medicina da Universidade de São Paulo (InCor HC FMUSP), a saber: o ATL modelo Apogee 800 Plus utilizado com um transdutor linear 11-5L40 (5 a $11 \mathrm{MHz}$ ) (ATL, 1996); o sistema portátil ACUSON modelo Cypress ${ }^{\mathrm{TM}}$, equipado com um transdutor 7L3 (5,22 MHz) (Siemens, 2002); o Philips/ATL modelo HDI 5000 com transdutor P4-2 (2 a 4 MHz) ou P5-3 (3 a 5 MHz); e o HP/Philips modelo Sonos 5500, com transdutor S3 (1 a $3 \mathrm{MHz}$ ) ou S4 (2 a $4 \mathrm{MHz})$.

\section{Aplicativo computacional}

O presente trabalho utilizou como base um aplicativo computacional desenvolvido por Pilon (2002) (Gutierrez et al., 2002), especializado para os cálculos da espessura da parede e do diâmetro arteriais a partir de imagens de ultrassonografia modo-B. Ao aplicativo foi acrescentado um novo módulo para os cálculos de

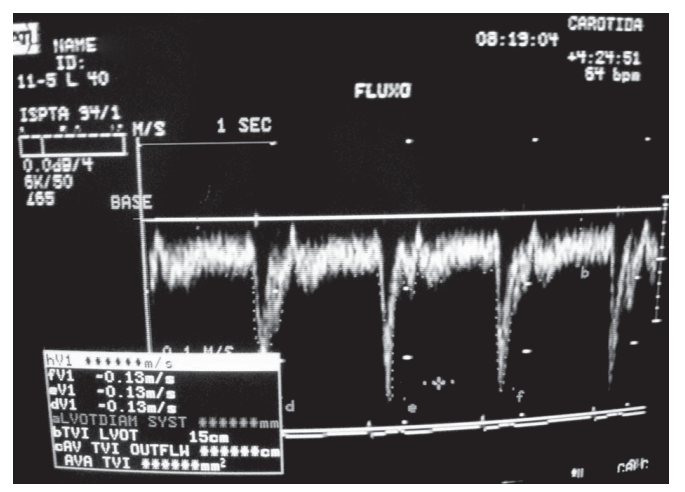

Figura 1. Tela típica de um sistema de ultrassonografia que mostra um gráfico da velocidade de sangue vascular em função do tempo e as correspondentes medições de amplitude e integral.

Figure 1. Typical screen of an ultrasound system showing vessel's blood velocity vs. time graphic and related amplitude and integral measurements. 
velocidade e fluxo de sangue em imagens adquiridas por ultrassonografia associada à técnica Doppler. A estrutura anterior foi aproveitada com a adoção do mesmo ambiente de programação: o Microsoft ${ }^{\circledR}$ Visual C++ (versão 6.0, 1998) complementado pela biblioteca de classes MFC.

\section{Metodologia proposta}

Nos itens seguintes são descritas as etapas de preparação, processamento de imagem e cálculos envolvidos nesta metodologia:

Imagem de entrada previamente convertida para tons de cinza (escala de 0 a 255);

Calibração dos eixos de velocidade (y) e tempo (x) para extrair as escalas da imagem em pixel $(\mathrm{m} / \mathrm{s})$ e pixel/s, respectivamente. Com o auxílio do mouse e tendo como referência as marcas de escala presentes no gráfico, o usuário desenha uma linha vertical e uma horizontal e, através de caixas de diálogo, atribui os tamanhos das linhas (pixels) nas grandezas correspondentes (m/s ou s). A Figura 2 ilustra a etapa de calibração do eixo " $y$ ";

Seleção de uma região de interesse (region of interest - ROI) retangular definida pelo usuário, também através do mouse, que contenha a envoltória e o eixo " $x$ " a serem detectados (Figura 2);

Aplicação de um filtro Gaussiano com $\sigma$ (desviopadrão $)=1$ pixel definido empiricamente para a redução de ruídos de alta frequência (Figura 3);

Detecção do eixo " $x$ " da variável de tempo: sabendo-se que este eixo é inserido na imagem, na direção horizontal, pelo próprio sistema de ultrassonografia e que, geralmente, é composto por pixels com pouca variação de intensidade entre eles, definem-se as funções:

$$
\begin{aligned}
& g(i, j)= \begin{cases}\frac{1}{|I(i, j)-I(i+1, j)|+1}, & I(i, j)>z \\
0, & I(i, j) \leq z\end{cases} \\
& f(j)=\sum_{i=0}^{m-2} g(i, j), \quad j \in\left[y_{\min }, y_{\max }\right]
\end{aligned}
$$

sendo:

- $I(i, j)$ a intensidade (tom de cinza) da imagem nas coordenadas $i$ e $j$;

- $\quad m$ a largura (em pixels) da imagem;

- $y_{\min }$ e $y_{\text {max }}$ respectivamente, as ordenadas das linhas superior e inferior que delimitam a ROI definida pelo usuário;

- $z=40$ um limiar pré-estabelecido empiricamente para desconsiderar a imagem de fundo do gráfico que, em geral, assume tonalidades mais escuras.

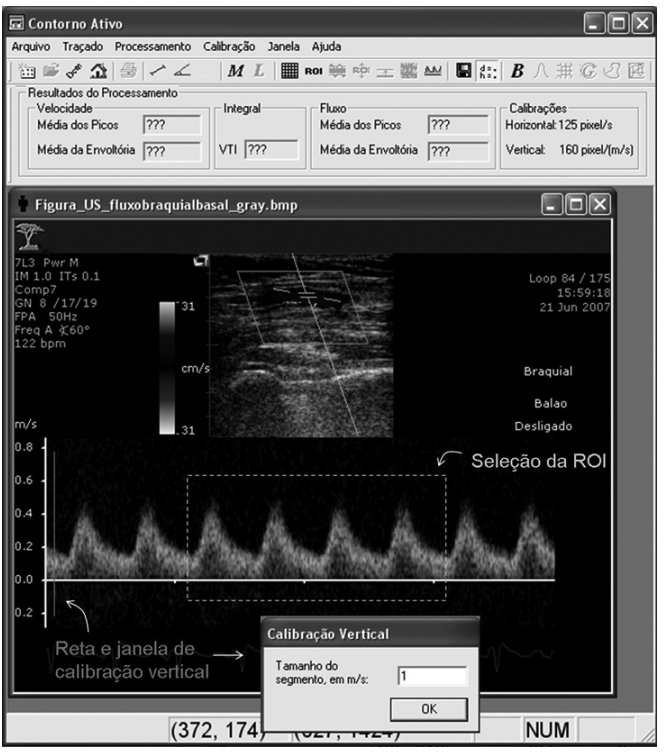

Figura 2. Etapas de calibração do eixo "y" (o usuário desenha a linha vertical e digita o tamanho correspondente $\mathrm{em} \mathrm{m} / \mathrm{s}$ na caixa de diálogo) e seleção da ROI (o usuário desenha um retângulo que contenha a envoltória e o eixo " $\mathrm{x}$ " a serem detectados).

Figure 2. Steps of ' $y$ ' axis calibration (the user draws a vertical line and input its size in $\mathrm{m} / \mathrm{s}$ in the dialog box) and ROI selection (the user draws a rectangle which contains the envelope and ' $x$ ' axis to be detected).

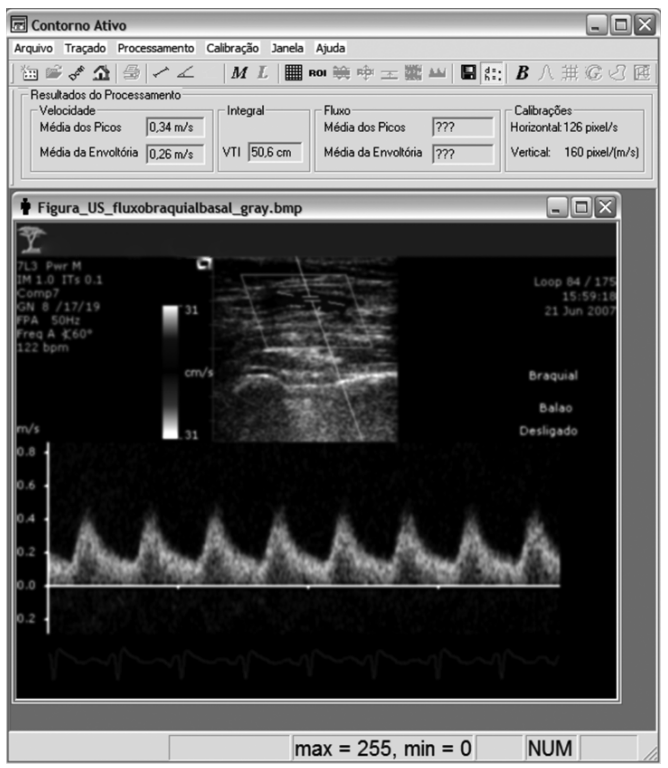

Figura 3. Resultado da aplicação de um filtro Gaussiano $(\sigma=1$ pixel $)$ para a redução de ruídos de alta frequência.

Figure 3. Result of applying a Gaussian filter ( $\sigma=1$ pixel) to attenuate high frequency noise.

A ordenada " $y$ " do eixo " $x$ " é, então, calculada pela expressão:

$y=\left\{j \in\left[y_{\min }, y_{\max }\right] \mid f(y)=\max f(j)\right\}$ 
Em suma, quanto menor a variação de intensidade de pixel ao longo de uma linha pertencente à ROI, maior será a soma cumulativa nesta linha dada por $f(j)$. O valor $j$ que maximiza esta função é atribuído à ordenada " $y$ " esperada do eixo " $x$ ". Este eixo será a referência, equivalente a $0 \mathrm{~m} / \mathrm{s}$, para o cálculo da velocidade (positiva ou negativa) após a detecção do contorno (superior ou inferior) da amplitude de velocidade (Figura 4);

Binarização com um limiar de tom de cinza (entre 0 e 255): o pixel assume o valor 255 ("ON") quando sua intensidade estiver acima do limiar ou o valor 0 ("OFF"), caso contrário (Figura 5). Conforme justificado mais adiante na seção "Resultados e Discussão", o valor do limiar mais apropriado e definido como padrão nesta aplicação é 40, mas pode ser configurado pelo usuário de acordo com o seu próprio critério, para melhor ajuste da envoltória;

Aplicação de um filtro Mediano na imagem binarizada usando um elemento estruturante de tamanho $3 \times 3$ pixels para a suavização das bordas e supressão de espúrios (Figura 6);

Detecção da envoltória da amplitude de velocidade: uma curva é inicializada na mesma posição da linha superior da ROI retangular. Em seguida, cada ponto desta curva é movido de cima para baixo até encontrar um pixel $\mathrm{ON}$ da imagem binarizada. Como resultado, esta curva se ajusta automaticamente ao contorno superior do gráfico de velocidade. De modo semelhante, outra curva é inicializada na linha inferior da ROI e se ajusta ao contorno inferior do gráfico. Porém, como em cada gráfico de velocidade normalmente é desejável apenas o contorno superior (velocidade positiva) ou o inferior (velocidade negativa), descartase automaticamente aquele que apresenta menor variação na amplitude (que comumente coincide com o próprio eixo “ $x$ ”), pelo cálculo da soma cumulativa dos módulos das diferenças de amplitude entre os pontos vizinhos ao longo do contorno (superior ou inferior) (Figura 4).

Entretanto, um dos sistemas utilizados para a aquisição das imagens de velocidade, o ATL Apogee, insere marcas de escala no interior do gráfico que se confundem com o espectro de velocidade pela semelhança da tonalidade e, portanto, devem ser tratadas como ruído neste processo. Para evitar detecções indesejáveis dos pixels ON destas marcas, introduziu-se uma etapa intermediária que utiliza a contagem de componentes conexos da imagem binária no esquema de 4-conectividade (vizinhos acima, abaixo, à esquerda e à direita) (Gonzalez e Woods, 2002). Assim, o deslocamento dos pontos das curvas superior e inferior até a envoltória desejada continua até que duas condições sejam satisfeitas: o pixel da imagem seja ON e a quantidade de componentes conexos com classificação $\mathrm{ON}$ seja maior ou igual a 110 pixels (valor determinado empiricamente). Portanto, pixels $\mathrm{ON}$ agrupados em pequena quantidade, como as marcas de escala, são desprezados na detecção da envoltória (Figura 7);
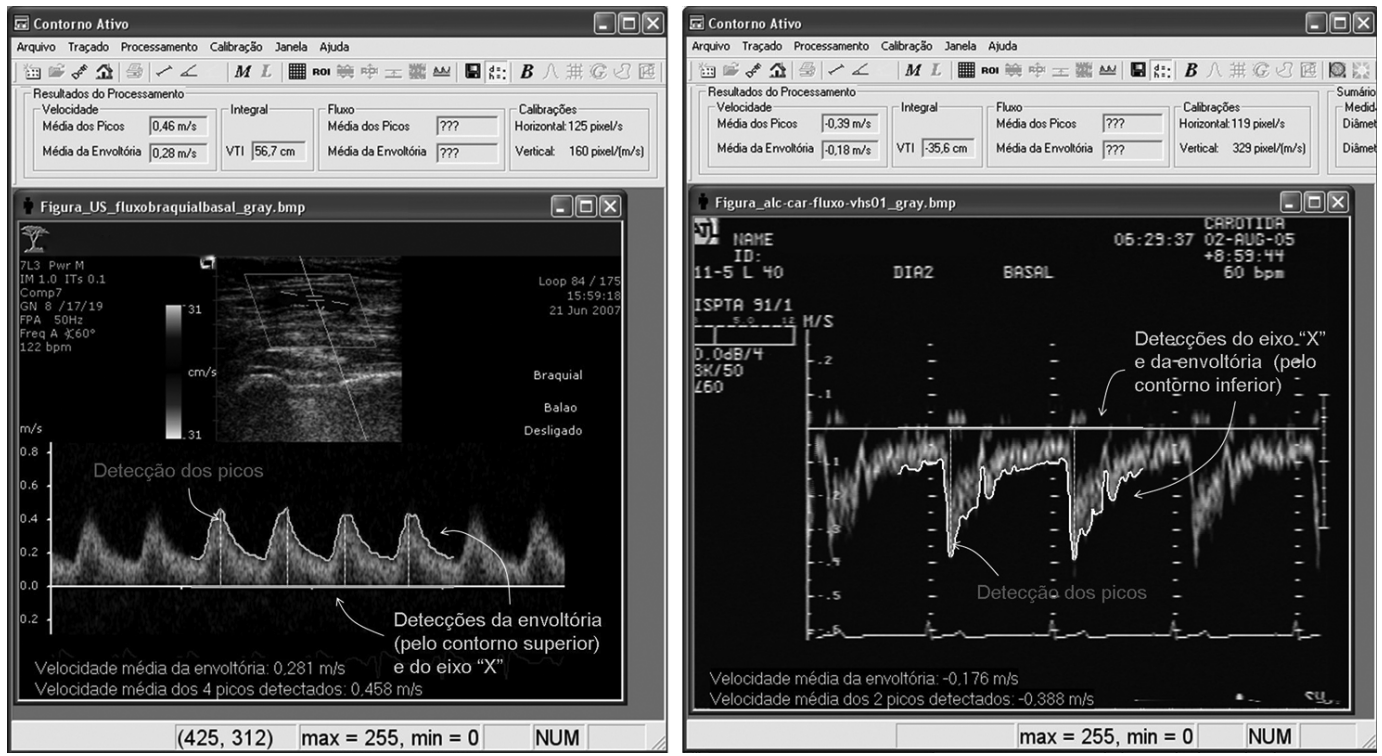

Figura 4. Detecções do eixo "x" (linha horizontal em $0 \mathrm{~m} / \mathrm{s}$ ), da envoltória do contorno superior (figura à esquerda), da envoltória do contorno inferior (figura à direita) e dos picos (indicados pelas linhas verticais).

Figure 4. Detection of the axis ' $x$ ' (horizontal line in $0 \mathrm{~m} / \mathrm{s}$ ), the superior contour envelope (figure to the left), the inferior contour envelope (figure to the right) and peaks (as indicated by vertical lines). 


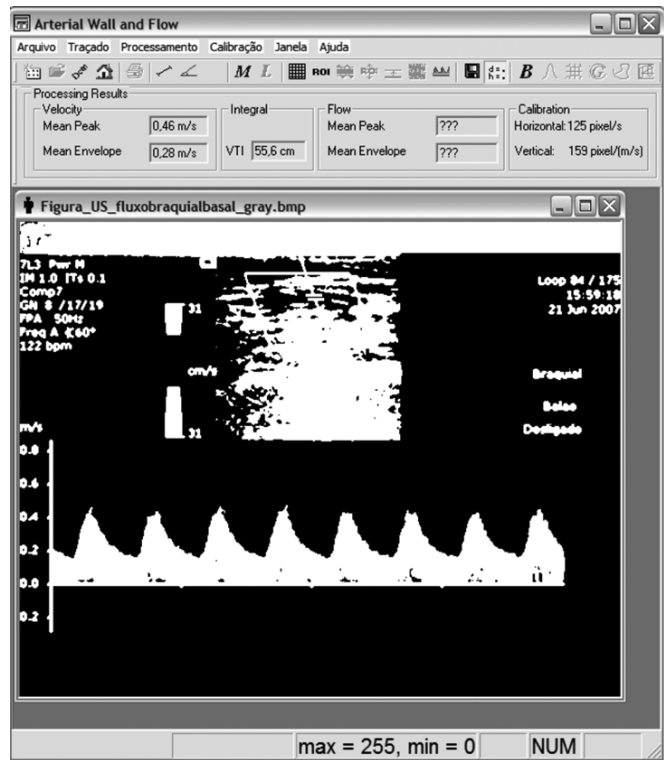

Figura 5. Binarização com um limiar padrão de tom de cinza igual a 40 . Figure 5. Binarization with a standard grayscale threshold of 40 .

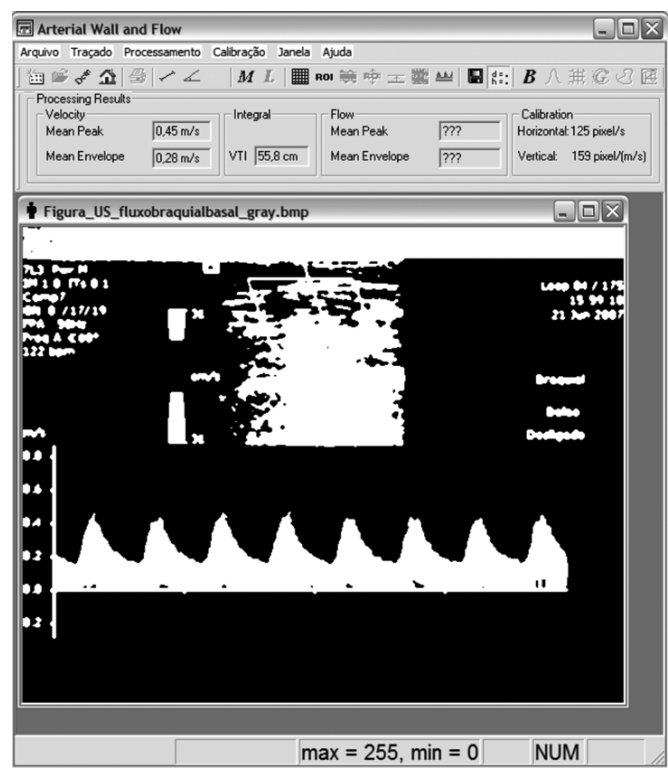

Figura 6. Resultado da aplicação de um filtro Mediano (tamanho: $3 \times 3$ pixels) para a suavização das bordas e supressão de espúrios. Figure 6. Result of applying a Median filter (size: $3 \times 3$ pixels) for edge smoothing and spurious suppression.

Detecção dos picos positivos ou negativos (Figura 4) com opção de escolha, através de uma caixa de diálogo, da distância mínima entre eles (valor padrão de 30 pixels) para evitar a detecção de picos intermediários indesejados. O método consiste em fazer uma varredura ponto a ponto, da esquerda para a direita, ao longo da curva da envoltória, e classificar

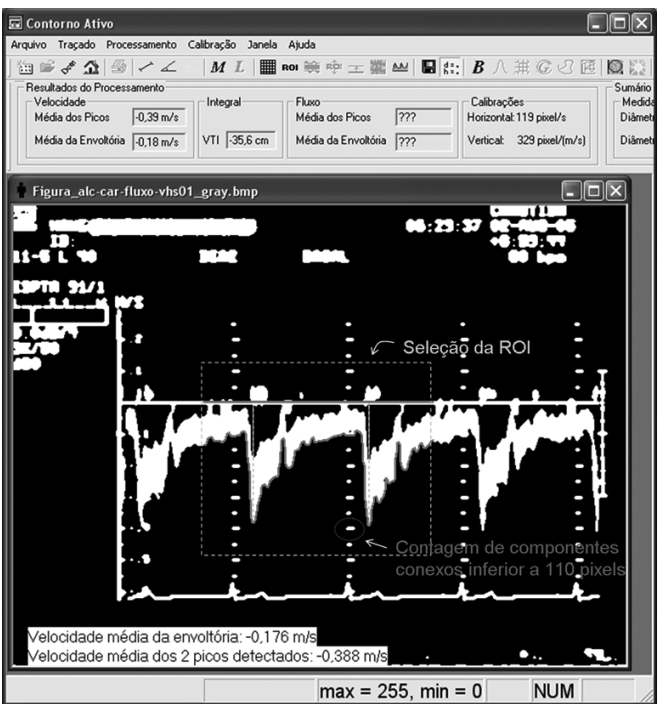

Figura 7. Contagem de componentes conexos na imagem binária para evitar a falsa detecção da envoltória.

Figure 7. Connected components counting in the binary image to avoid false detection of the envelope.

cada ponto da curva como pico se, à sua direita $\mathrm{e}$ dentro da distância estabelecida na caixa de diálogo, não houver outro ponto com maior amplitude. Um detalhe adicional é a desconsideração dos primeiros pontos (na parte esquerda da curva) contidos na janela definida pelo mesmo parâmetro de distância para evitar a falsa detecção de picos neste intervalo.

O cálculo da velocidade média dos picos detectados, da velocidade média da envoltória e da VTI, é realizado conforme as expressões:

Veloc. média dos picos $[\mathrm{m} / \mathrm{s}]=\frac{1}{P \cdot Y C a l} \sum_{p=1}^{P} A_{p}$

Veloc. média da envoltória $[\mathrm{m} / \mathrm{s}]=\frac{1}{N \cdot Y C a l} \sum_{n=1}^{N} A_{n}$

$\operatorname{VTI}[\mathrm{cm}]=\frac{100}{X C a l \cdot Y C a l} \sum_{n=1}^{N} A_{n}$

sendo:

- $A$ a amplitude, em pixels, de cada ponto da envoltória;

- $P$ o total de picos detectados;

- $\quad N$ o total de pontos da envoltória;

- YCal a escala de calibração vertical, em pixels/ $(\mathrm{m} / \mathrm{s})$;

- XCal a escala de calibração horizontal em pixels/s.

As estimativas do fluxo médio dos picos e do fluxo médio da envoltória são realizadas, considerando o diâmetro da luz máximo e mínimo e uma geometria 
circular para a seção transversal da artéria, conforme Expressões 8 e 9 .

Fluxo médio dos picos $[1 / \mathrm{min}]=$ $0,06 \cdot \bar{V}_{P} \cdot \frac{\pi D_{\max }^{2}}{4}$

Fluxo médio da envoltória $[1 / \mathrm{min}]=$

$$
0,06 \cdot \bar{V}_{N} \cdot \frac{\pi\left(D_{\max }+D_{\min }\right)^{2}}{16}
$$

sendo:

- $\overline{\mathrm{V}}_{\mathrm{P}}$ a velocidade média dos picos $[\mathrm{m} / \mathrm{s}]$ calculada no item anterior;

- $\overline{\mathrm{V}}_{\mathrm{N}}$ a velocidade média da envoltória $[\mathrm{m} / \mathrm{s}]$ calculada no item anterior;

- $D_{\max }$ e $D_{\min }$ respectivamente, as médias dos diâmetros máximo e mínimo da luz [mm]. Podem ser obtidas durante uma etapa preliminar de processamento de imagens arteriais (Gutierrez et al., 2002; Pilon, 2002).

\section{Composição das amostras}

Para a validação da metodologia, de cada gráfico do espectro de velocidades foram medidas de 1 a 4 velocidades de pico sistólico e até duas VTIs ao longo dos ciclos cardíacos. As imagens estavam distribuídas conforme a Tabela 1 que apresenta também o subtotal das amostras para cada tipo de imagem. No total foram utilizadas 102 amostras de velocidade de pico e 75 de VTI.

Outras medições como o fluxo e a velocidade média não foram avaliadas diretamente neste trabalho. Porém, suas análises estão contempladas indiretamente pelas suas relações com a metodologia de detecção da envoltória e com o cálculo da velocidade de pico.

As imagens Doppler das artérias carótida e braquial foram adquiridas com o Apogee 800 Plus e com o Cypress $^{\mathrm{TM}}$ e as medições através destes sistemas foram realizadas por uma única operadora treinada. As de ecocardiografia foram adquiridas com o HDI 5000 e com o Sonos 5500; elas estavam disponíveis na base de dados de produção do InCor e as medições foram

Tabela 1. Composição das amostras utilizadas para a validação da metodologia.

Table 1. Composition of samples used to validate the methodology.

\begin{tabular}{|c|c|c|c|}
\hline \multirow{2}{*}{ Imagens } & \multirow{2}{*}{$\begin{array}{l}\text { Quantidade de } \\
\text { imagens }\end{array}$} & \multicolumn{2}{|c|}{ Quantidade de amostras } \\
\hline & & Velocidade de pico & VTI \\
\hline Artéria carótida comum & 30 & 39 & 31 \\
\hline Artéria braquial na condição basal & 23 & 35 & 24 \\
\hline $\begin{array}{l}\text { Artéria braquial na situação de hiperemia reativa } \\
\text { após procedimento de oclusão }\end{array}$ & 10 & 15 & 15 \\
\hline Ecocardiografia & 11 & 13 & 5 \\
\hline Total & 74 & 102 & 75 \\
\hline
\end{tabular}
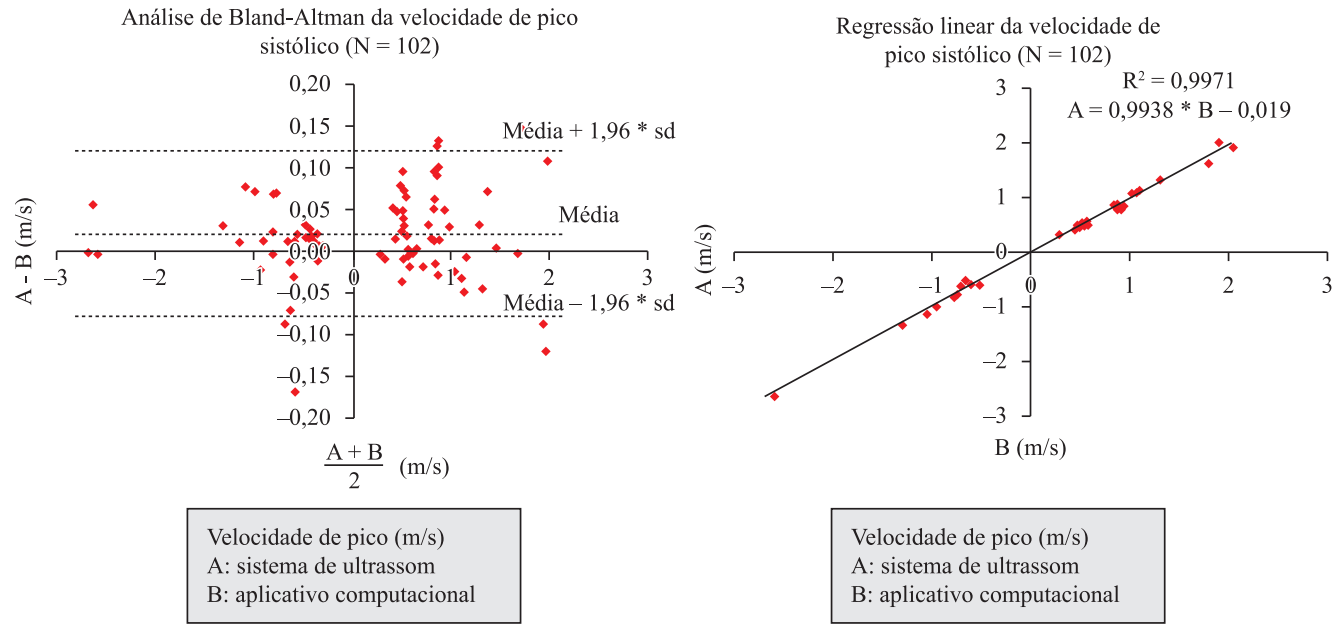

Figura 8. Análises de Bland-Altman e Regressão Linear da velocidade de pico sistólico medida pelo sistema de ultrassonografia e pela metodologia proposta.

Figure 8. Bland-Altman's and Linear Regression analysis of the systolic peak velocity measured by the ultrasound system and by the proposed methodology. 
Tabela 2. Resumo dos cálculos estatísticos da velocidade de pico sistólico: diferença média (d), desvio padrão (sd), coeficiente de correlação de Pearson $\left(\mathrm{r}_{\mathrm{AB}}\right)$ e equação da reta de regressão. Table 2. Summary of systolic peak velocity statistics: mean difference (d), standard deviation (sd), Pearson's correlation coefficient $\left(r_{A B}\right)$ and linear equation.

\begin{tabular}{cccc}
\hline & \multicolumn{3}{c}{ Velocidade de pico sistólico (N=102) } \\
\hline $\begin{array}{c}\mathbf{d} \\
(\mathbf{m} / \mathbf{s})\end{array}$ & $\begin{array}{c}\mathbf{s d} \\
(\mathbf{m} / \mathbf{s})\end{array}$ & $\begin{array}{c}\mathbf{r}_{\mathrm{AB}} \\
(\mathbf{p}<\mathbf{0 , 0 1})\end{array}$ & $\begin{array}{c}\text { Equação de regressão } \\
\text { linear }\end{array}$ \\
\hline 0,02 & 0,05 & 0,9985 & $\mathrm{~A}=0,9938 * \mathrm{~B}-0,0190$ \\
\hline
\end{tabular}

Análise de Bland-Altman da VTI $(\mathrm{N}=75)$
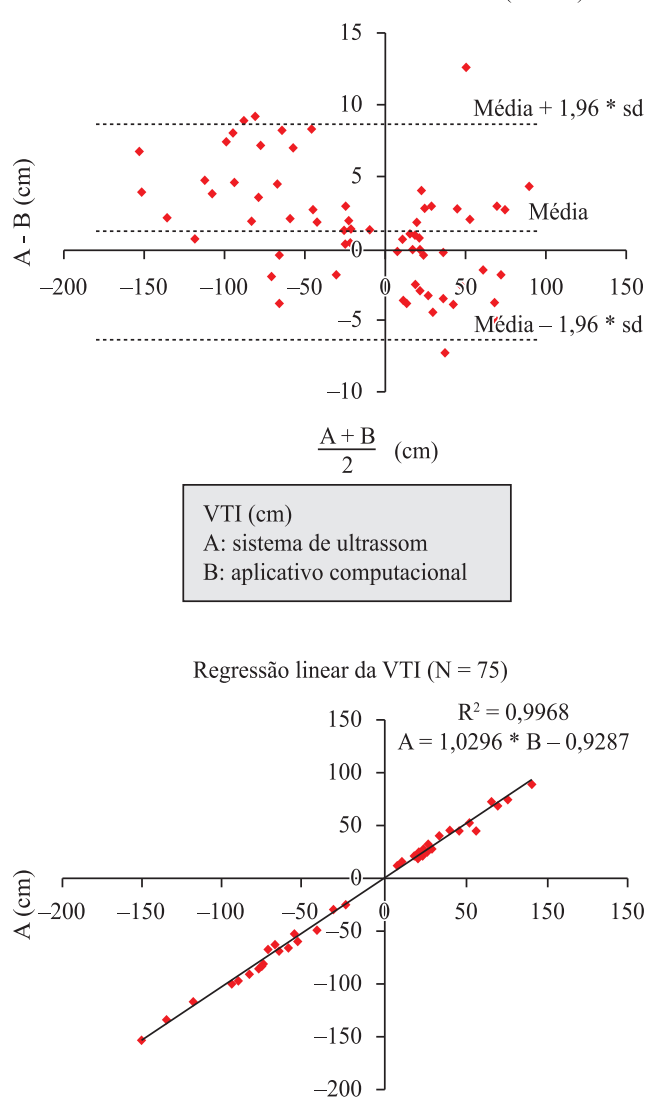

VTI $(\mathrm{cm})$

A: sistema de ultrassom

B: aplicativo computacional

Figura 9. Análises de Bland-Altman e Regressão Linear da VTI medida pelo sistema de ultrassonografia e pela metodologia proposta. Figure 9. Bland-Altman's and Linear Regression analysis of the VTI measured by the ultrasound system and by the proposed methodology.

realizadas por clínicos distintos, não identificados. As mesmas amostras foram, então, processadas de forma independente pelo novo módulo computacional com os parâmetros mantidos constantes para todas as imagens.
Tabela 3. Resumo dos cálculos estatísticos da VTI: diferença média (d), desvio padrão (sd), coeficiente de correlação de Pearson $\left(\mathrm{r}_{\mathrm{AB}}\right)$ e equação da reta de regressão.

Table 3. Summary of VTI statistics: mean difference (d), standard deviation (sd), Pearson's correlation coefficient $\left(r_{A B}\right)$ and linear equation.

\begin{tabular}{cccc}
\hline \multicolumn{3}{c}{ VTI $(\mathbf{N}=\mathbf{7 5})$} \\
\hline $\begin{array}{c}\mathbf{d} \\
(\mathbf{c m})\end{array}$ & $\begin{array}{c}\mathbf{s d} \\
(\mathbf{c m})\end{array}$ & $\begin{array}{c}\mathbf{r}_{\mathrm{AB}} \\
(\mathbf{p}<\mathbf{0 , 0 1})\end{array}$ & $\begin{array}{c}\text { Equação de regressão } \\
\text { linear }\end{array}$ \\
\hline 1,25 & 3,86 & 0,9984 & $\mathrm{~A}=1,030 * \mathrm{~B}-0,9287$ \\
\hline
\end{tabular}

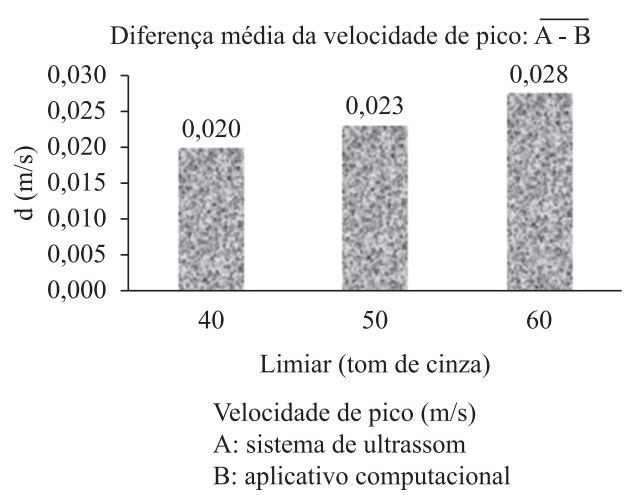

Figura 10. Diferença média das velocidades de pico entre os sistemas de ultrassonografia e a metodologia proposta com os limiares de tons de cinza de 40, 50 e 60.

Figure 10. Mean difference of the peak velocities between the ultrasound systems and the proposed methodology with grayscale thresholds of 40, 50 and 60.

\section{Resultados e Discussão}

Conforme o procedimento utilizado, as velocidades de pico medidas na artéria carótida foram negativas, com um valor médio das amostras de $-0,59 \mathrm{~m} / \mathrm{s}$. Na artéria braquial, tanto na condição basal como na resposta de hiperemia reativa, as velocidades de pico foram positivas, com as respectivas médias de 0,63 e $1,18 \mathrm{~m} / \mathrm{s}$. Finalmente, nas imagens de ecocardiografia as medições realizadas pelos clínicos foram negativas ou positivas, com uma média dos valores absolutos de $1,48 \mathrm{~m} / \mathrm{s}$.

De modo análogo, os sinais (positivos ou negativos) das VTIs dependeram do tipo de exame realizado; contudo, não houve uma padronização do número de ciclos cardíacos para a medição desta grandeza. As amostras variaram no intervalo de $-150 \mathrm{~cm}$ a $91 \mathrm{~cm}$.

Os gráficos da Figura 8 apresentam análises comparativas pelo método de Bland-Altman (Bland e Altman, 1986) e pela regressão linear da velocidade de pico sistólico para o total de 102 amostras. A grandeza " $A$ " corresponde às medições realizadas através dos sistemas de ultrassonografia e a grandeza " $B$ " aos resultados obtidos pela metodologia proposta. 
A diferença média (calculada como: $d=\overline{\mathrm{A}-\mathrm{B}}$ ) e o desvio-padrão (sd) são mostrados na Tabela 2 que inclui, também, o coeficiente de correlação de Pearson $\left(\mathrm{r}_{\mathrm{AB}}\right)($ Costa Neto, 1977) e a equação da reta de regressão.

Tanto a diferença média quanto o desvio-padrão estimados foram muito baixos, como desejado, indicando que a metodologia proposta pode ser validada. No trabalho de Tschirren et al. (2001), que propôs outra metodologia com objetivo semelhante, a magnitude da diferença média e o desvio-padrão da velocidade de pico sistólico foram maiores: $0,40 \mathrm{~m} / \mathrm{s}$ e $1,48 \mathrm{~m} / \mathrm{s}$, respectivamente. Há que se considerar, porém, que, naquele trabalho, os dados
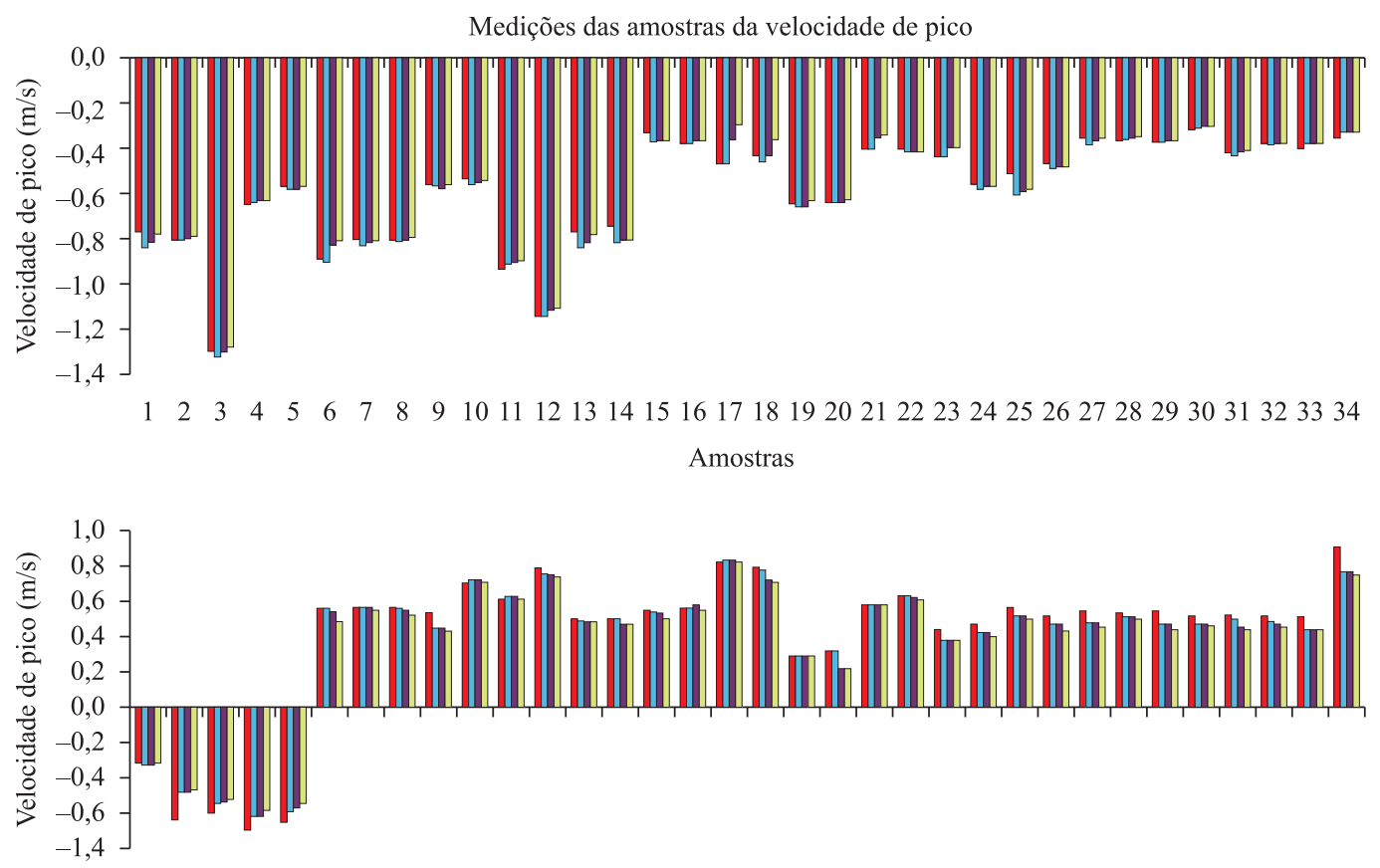

35363738394041424344454647484950515253545556575859606162636465666768

Amostras

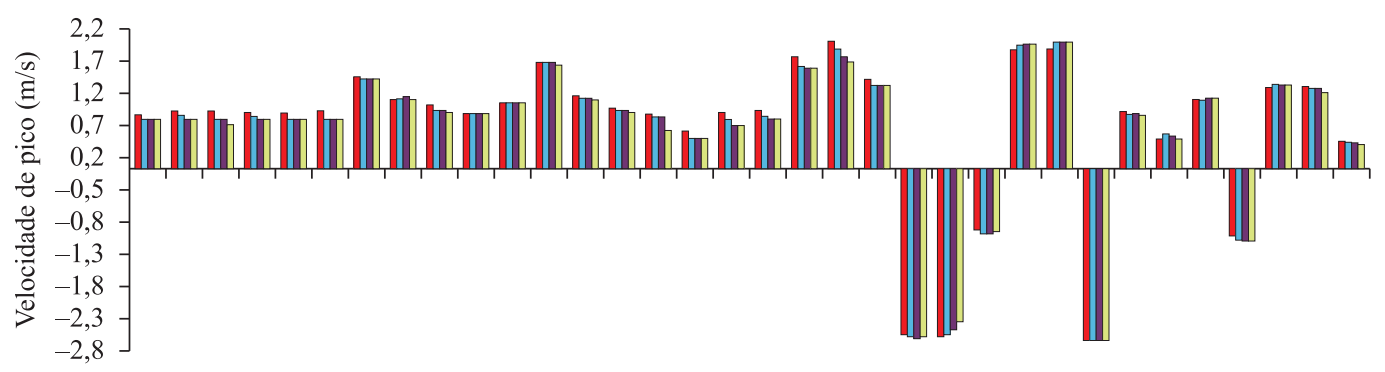

69707172737475767778798081828384858687888990919293949596979899100101102

Amostras

\begin{tabular}{|ll|}
\hline$\square$ Medição "A" & $\square$ Medição "B" com limiar 40 \\
$\square$ Medição "B" com limiar 50 & $\square$ Medição "B" com limiar 60 \\
\hline
\end{tabular}

Figura 11. Representação gráfica das 102 amostras das velocidades de pico medidas com os sistemas de ultrassonografia (Medição "A") e com o aplicativo computacional (Medição "B”), utilizando três limiares de níveis de cinza: 40, 50 e 60. As amostras são da artéria carótida comum (amostras 1 a 39), da artéria braquial na condição basal (amostras 40 a 74), da artéria braquial na situação de hiperemia reativa (amostras 75 a 89 ) e de ecocardiografia (amostras 90 a 102).

Figure 11. Graphic representation of 102 peak velocity samples measured by the ultrasound systems (Measurement 'A') and by the computational tool (Measurement 'B'), using three grayscale thresholds: 40, 50 and 60. The samples are of common carotid artery (samples 1 to 39), brachial artery under basal condition (samples 40 to 74), brachial arteries in the reactive hyperemic response (samples 75 to 89) and echocardiographic exams (samples 90 to 102). 


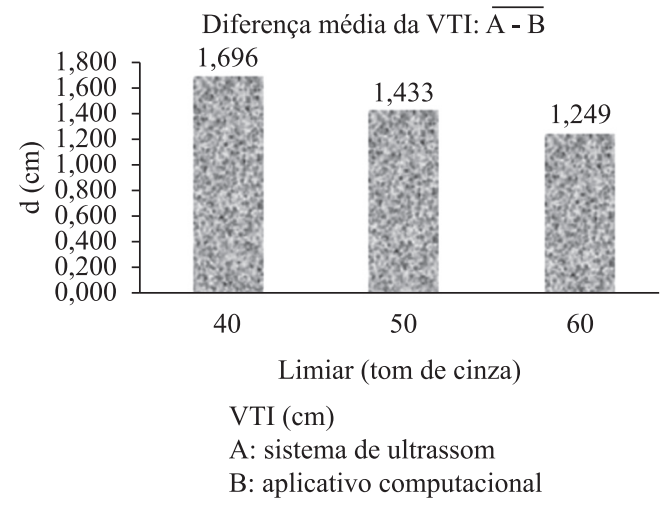

Figura 12. Diferença média das VTIs entre os sistemas de ultrassonografia e a metodologia proposta com os limiares de tons de cinza de 40, 50 e 60.

Figure 12. Mean difference of the VTIS between the ultrasound systems and the proposed methodology with grayscale thresholds of 40,50 and 60 . eram provenientes de um estudo de dilatação da artéria braquial, geralmente da ordem de grandeza 10 vezes superior às das amostras do presente estudo.

A expressão da reta de regressão, o coeficiente de correlação próximo a 1 e a disposição dos dados no gráfico da Figura 8 corroboram a confiabilidade desta metodologia.

Como na análise da velocidade de pico, o método de Bland-Altman e a regressão linear aplicados à VTI resultaram na Figura 9 para o total de 75 amostras. Os valores calculados da diferença média, do desviopadrão, do coeficiente de correlação e da equação da reta de regressão linear estão dispostos na Tabela 3. Tschirren et al. (2001) obtiveram uma diferença média e um desvio-padrão maiores para a VTI: $7 \mathrm{~cm}$ e $122 \mathrm{~cm}$, respectivamente.

O gráfico da Figura 10 indica que a menor diferença média (d) da velocidade de pico foi alcançada com o limiar de 40 , quando comparada com as dos limiares de 50 e 60 . Através de gráficos de barras, a Figura 11

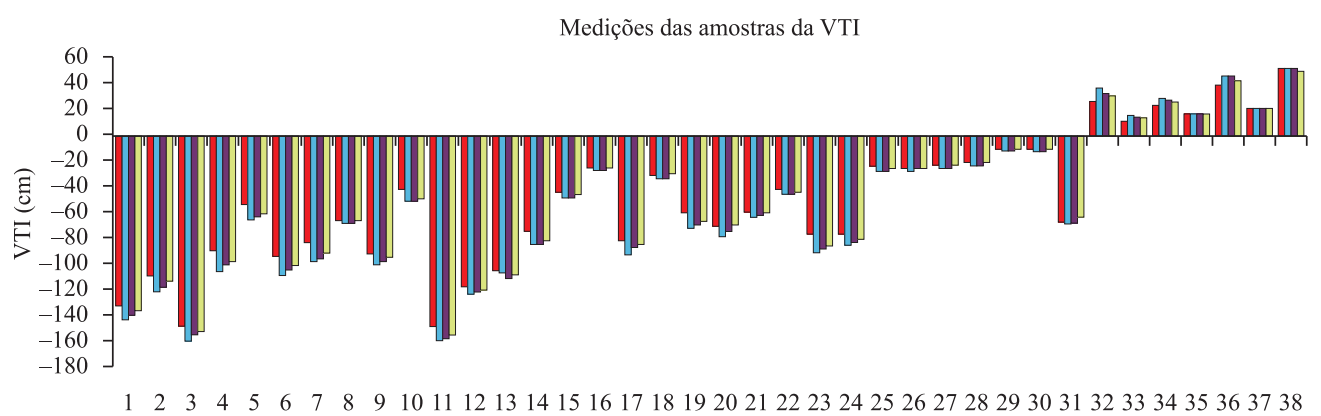

Amostra

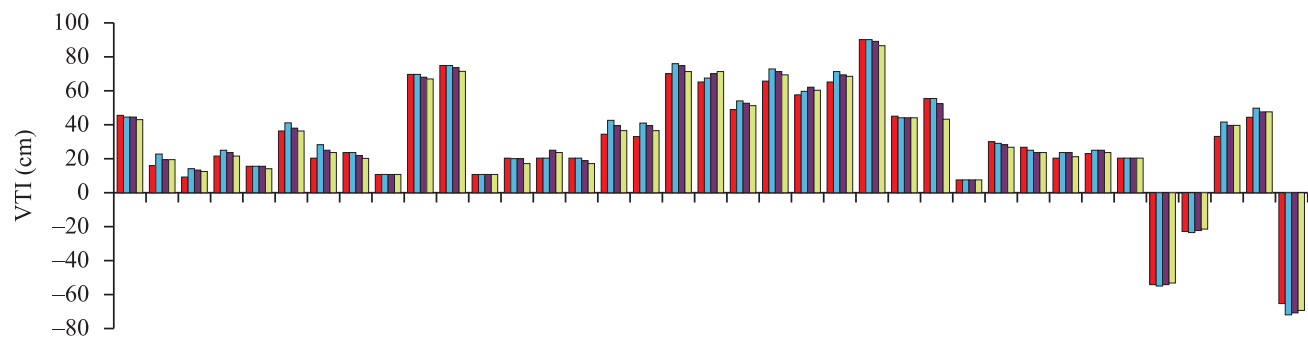

39404142434445464748495051525354555657585960616263646566676869707172737475

Amostra

\begin{tabular}{|ll|}
\hline$\square$ Medição "A" & $\square$ Medição "B" com limiar 40 \\
$\square$ Medição "B" com limiar 50 & $\square$ Medição "B" com limiar 60 \\
\hline
\end{tabular}

Figura 13. Representação gráfica das 75 amostras das VTIs medidas com os sistemas de ultrassonografia (Medição “A”) e com o aplicativo computacional (Medição "B”), utilizando três limiares de níveis de cinza: 40, 50 e 60. As amostras são da artéria carótida comum (amostras 1 a 31), da artéria braquial na condição basal (amostras 32 a 55), da artéria braquial na situação de hiperemia reativa (amostras 56 a 70 ) e de ecocardiografia (amostras 71 a 75).

Figure 13. Graphic representation of 75 VTI samples measured by the ultrasound systems (Measurement 'A') and by the computational tool (Measurement ' $B$ '), using three grayscale thresholds: 40, 50 and 60. The samples are of common carotid artery (samples 1 to 31), brachial artery under basal condition (samples 32 to 55), brachial arteries in the reactive hyperemic response (samples 56 to 70) and echocardiographic exams (samples 71 to 75 ). 
mostra todos os valores das medições da velocidade de pico, tanto com os sistemas de ultrassonografia (Medição "A"), quanto com a metodologia proposta (Medição "B"), utilizando os três limiares. Ensaios com o limiar de 30 também foram realizados, mas logo, desconsiderados, uma vez que para várias amostras os resultados visuais já mostravam que a detecção da envoltória era afetada pelo ruído na imagem.

Cabe observar que os resultados da VTI foram alcançados com um limiar de conversão da imagem de tons de cinza para binária (vide item "binarização" da subseção "Metodologia proposta") igual a 60 . A alteração em relação ao limiar padrão de 40 utilizado na extração da velocidade de pico sistólico foi motivada pelos resultados mais próximos em relação à medição através do sistema de ultrassonografia, como mostra o gráfico da Figura 12. Por exemplo, com o limiar de 40, na análise comparativa entre os dois métodos a diferença média e o desvio-padrão da VTI foram maiores: $1,70 \mathrm{~cm}$ e $6,78 \mathrm{~cm}$, respectivamente. Apesar destes dados, não se pode afirmar que o limiar de 60 é mais apropriado que o de 40, pois o procedimento manual do operador do equipamento de ultrassonografia também está sujeito a erros e aproximações.

A Figura 13 apresenta, graficamente, todos os valores das medições da VTI, tanto com os sistemas de ultrassonografia, quanto com a metodologia proposta utilizando os limiares de 40, 50 e 60. Ensaios com o limiar de 70 foram desconsiderados porque, para várias amostras, a detecção ocorria claramente no interior da imagem do espectro e não mais na envoltória.

Pela observação visual das envoltórias traçadas sobre os gráficos de velocidade nota-se que, pela metodologia proposta (Figura 4), a envoltória é mais rica em detalhes que na obtida pelo sistema de ultrassonografia (Figura 1), pois, neste último, o operador não percebe ou, simplesmente, desconsidera pequenas variações de brilho da imagem. Isto ocorre, em parte, porque, para uma aproximação mais refinada, exige-se uma manipulação mais cuidadosa do trackball para desenhar o contorno. Assim, este procedimento, que é altamente dependente do operador, caracteriza-se pela subjetividade e difícil reprodutibilidade.

O aplicativo computacional desenvolvido neste trabalho possibilita que, através de uma caixa de diálogo, o usuário altere o limiar de binarização do gráfico de velocidade. Este ajuste atua diretamente na detecção da envoltória, adequando-se ao critério do usuário, pois o seu incremento faz com que todo o contorno seja deslocado para o interior da imagem do espectro correspondente ao gráfico de velocidade $\mathrm{e}$, analogamente, o decremento do limiar faz com que todo o contorno seja deslocado para o exterior da imagem do espectro. Os resultados visuais associados aos estatísticos foram determinantes na adoção do limiar de 40 para a conversão binária como o valor padrão da caixa de diálogo.

\section{Conclusão}

Utilizando uma diversidade de imagens Doppler das artérias carótida comum e braquial e de exames ecocardiográficos provenientes de quatro modelos distintos de sistemas comerciais de ultrassonografia, a metodologia implementada neste trabalho para os cálculos de velocidade e VTI foi validada pela análise de Bland-Altman e pelos coeficientes de correlação. A análise visual também confirmou que a deteç̧ão da envoltória do espectro do gráfico de velocidade é bastante satisfatória.

Como produto deste trabalho, um aplicativo executável em computadores pessoais pode ser disponibilizado e facilmente utilizado pelos clínicos que tenham interesse em estudar possíveis relações entre a velocidade ou fluxo sanguíneo e doenças cardiovasculares, entre outras aplicações. A distribuição e o controle de cópias do arquivo executável estão sob a coordenação do Serviço de Informática do InCor HC FMUSP.

A combinação da interface gráfica amigável para o usuário e a característica semiautomática desta ferramenta computacional elimina a delineação manual da envoltória e, consequentemente, proporciona as seguintes vantagens: redução do tempo operacional, redução da subjetividade correspondente à simplificação ou não percepção do operador para acompanhar as variações de brilho da imagem, e aumento do grau de reprodutibilidade dos resultados.

\section{Agradecimentos}

À Coordenação de Aperfeiçoamento de Pessoal de Nível Superior (CAPES) e à Fundação de Apoio à Universidade de São Paulo (FUSP) pelos recursos financeiros que viabilizaram as atividades deste projeto.

Ao InCor HC FMUSP, especialmente à área de Pesquisa e Desenvolvimento do Serviço de Informática e ao Laboratório de Investigação Clínica da Unidade de Terapia Intensiva pelas contribuições técnicas e operacionais.

\section{Referências}

ATL. Apogee 800 Plus ultrasound system reference manual, 1996. Rev. A 9/96, n. 4703-0019-03.

BAMBER, J. C.; TRISTAM, M. Diagnostic ultrasound In: WEBB, S. (Ed.). The physics of medical imaging. London: IOP Publishing, 1993. cap. 7, 633 p. 
BLAND, J. M.; ALTMAN, D. G. Statistical methods for assessing agreement between two methods of clinical measurement. The Lancet, v. 327, n. 8476, p. 307-310, 1986. http://dx.doi.org/10.1016/S0140-6736(86)90837-8

CARVALHO, R. T.; VIEIRA, M. L. C.; ROMANO, A.; KOPEL, L.; LAGE, S. G. Exercício resistido na avaliação da disfunção endotelial na insuficiência cardíaca. Arquivos Brasileiros de Cardiologia, v. 86, n. 6, p. 459-465, 2006.

CORRETTI, M. C.; ANDERSON, T. J.; BENJAMIN, E. J.; CELERMAJER, D.; CHARBONNEAU, F.; CREAGER, M. A.; DEANFIELD, J.; DREXLER, H.; GERHARDHERMAN, M.; HERRINGTON, D.; VALLANCE, P.; VITA, J.; VOGEL, R. Guidelines for the ultrasound assessment of endothelial-dependent flow-mediated vasodilation of the brachial artery: A report of the International Brachial Artery Reactivity Task Force. Journal of the American College of Cardiology, v. 39, n. 2, p. 257-265, 2002. http://dx.doi.org/10.1016/S07351097(01)01746-6

COSTA NETO, P. L. O. Estatística. Edgard Blücher, 1977. 264 p.

GERHARD-HERMAN, M.; GARDIN, J. M.; JAFF, M.; MOHLER, E.; ROMAN, M.; NAQVI, T. Z. Guidelines for noninvasive vascular laboratory testing: a report from the American Society of Echocardiography and the Society for Vascular Medicine and Biology. Vascular Medicine, v. 11, n. 3, p. 183-200, 2006. PMid:17288127. http://dx.doi. org/10.1177/1358863x06070516

GONZALEZ, R. C.; WOODS, R. E. Digital image processing. 2th ed. Upper Saddle River: Prentice Hall, 2002. 793 p.

GUTIERREZ, M. A.; PILON, P. E.; LAGE, S. G.; KOPEL, L.; CARVALHO, R. T.; FURUIE, S. S. Automatic measurement of carotid diameter and wall thickness in ultrasound images. In: COMPUTERS IN CARDIOLOGY, 2002, Memphis. Proceedings... Memphis, 2002. p. 359-362.
HOSKINS, P. R. Measurement of arterial blood-flow by Doppler ultrasound. Clinical Physics and Physiological Measurement, v. 11, n. 1, p. 1-26, 1990. PMid:2182271. http://dx.doi.org/10.1088/0143-0815/11/1/001

LAGE, S. G.; KOPEL, L.; MEDEIROS, C. C.; CARVALHO, R. T.; CREAGER, M. A. Angiotensin II contributes to arterial compliance in congestive heart failure. American Journal of Physiology - Heart and Circulatory Physiology, v. 283, n. 4, p. H1424-H1429, 2002.

NICHOLS, W.; O'ROURKE, M. Doppler ultrasound for arterial blood flow measurement. In: McDonald's blood flow in arteries: theoretic, experimental and clinical principles. 3th ed. Malvern, Philadelphia: Lea \& Febiger, 1990. cap. 7. 456 p.

PILON, P. E. Método para a deteç̧ão automática de diâmetro de artérias em imagens de ultra-som. 2002. 73 f. Dissertação (Mestrado em Engenharia Elétrica)-Escola Politécnica, Universidade de São Paulo, São Paulo, 2002.

ROUTH, H. F. Doppler ultrasound: the ability to measure and image blood flow. IEEE Engineering in Medicine and Biology Magazine, v. 15, n. 6, p. 31-40, 1996. http://dx.doi.org/10.1109/51.544510

SIEMENS MEDICAL SOLUTIONS USA, INC. ACUSON Cypress $^{\mathrm{TM}}$ System Operator's Manual. 2002. Rev. A, Document, n. 08267349.

TSCHIRREN, J.; LAUER, R. M.; SONKA, M. Automated analysis of Doppler ultrasound velocity flow diagrams. IEEE Transactions on Medical Imaging, v. 20, n. 12 , p. 1422-1425, 2001. PMid:11811841. http://dx.doi. org/10.1109/42.974936

YAO, J. S. T. Noninvasive studies of peripheral vascular disease (ch. 7). In: HOBSON, R. W.; WILSON, S. E.; VEITH, F. J. (Eds.). Vascular surgery: principles and practice. 3 th ed., rev. exp. Marcel Dekker, Inc., 2004. 1600 p.

\section{Autores}

\section{Mauricio Higa, Paulo Eduardo Pilon}

Escola Politécnica / USP, Av. Dr. Enéas de Carvalho Aguiar, 44, CEP 05403-000, São Paulo, SP, Brasil

\section{Sílvia Helena Gelás Lage}

Unidade de Terapia Intensiva Clínica do Instituto do Coração / HC FMUSP,

Av. Dr. Enéas de Carvalho Aguiar, 44, CEP 05403-000, São Paulo, SP, Brasil

\section{Marco Antonio Gutierrez}

Serviço de Informática do Instituto do Coração / HC FMUSP, Escola Politécnica / USP,

Av. Dr. Enéas de Carvalho Aguiar, 44, CEP 05403-000, São Paulo, SP, Brasil 